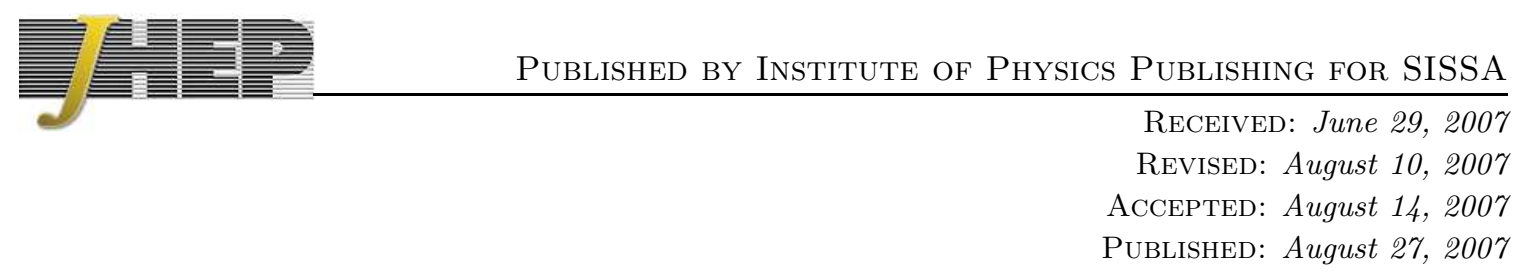

\title{
Ehlers symmetry at the next derivative order
}

\author{
Claudia Colonnello and Axel Kleinschmidt \\ Max Planck Institute for Gravitational Physics, Albert Einstein Institute, \\ Am Mühlenberg 1, 14476 Potsdam, Germany \\ E-mail: Claudia.Colonnello@aei.mpg.de, axel.kleinschmidt@aei.mpg.de
}

\begin{abstract}
We analyse four-dimensional gravity in the presence of general curvature squared corrections and show that Ehlers' $\mathrm{SL}(2, \mathbb{R})$ symmetry, which appears in the reduction of standard gravity to three dimensions, is preserved by the correction terms. The mechanism allowing this is a correction of the $\mathrm{SL}(2, \mathbb{R})$ transformation laws which resolves problems with the different scaling behaviour of various terms occurring in the reduction.
\end{abstract}

KEYwords: String Duality, Global Symmetries, Supergravity Models. 


\section{Contents}

1. Introduction 1

2. $R^{2}$ corrected action in $(3+1)$ dimensions 2

3. The reduced corrected action 3

4. Corrected $\operatorname{SL}(2, \mathbb{R})$ transformations 7

5. Outlook 8

A. Reduction of curvature tensors 9

\section{Introduction}

Supergravity theories, when compactified on tori, exhibit so-called hidden symmetries which extend the naïve torus symmetries [1- [3]. In the case of pure gravity in $(3+n)$ dimensions reduced on an $n$-torus, there is a hidden $\mathrm{SL}(n+1) / \mathrm{SO}(n+1)$ symmetry which occurs when dualising the field strengths of the Kaluza-Klein vectors obtained in the dimensional reduction to gradients of scalar fields in the three remaining space-time dimensions. This was first observed in the case of $(3+1)$ dimensions a long time ago and the enhanced symmetry $\operatorname{SL}(2, \mathbb{R})$ is known as Ehlers symmetry [4]. Many supergravity theories arise as the low energy limit $\left(\alpha^{\prime} \rightarrow 0\right)$ of string theory. String theory predicts in such cases also corrections to the two-derivative supergravity actions. These corrections are a double expansion in the string coupling $g_{s}$ and the squared string scale $\alpha^{\prime}=\ell_{s}^{2}$, potentially with non-perturbative contributions. Since $\alpha^{\prime}$ is dimensionful, higher powers of $\alpha^{\prime}$ must be accompanied by a higher number of derivatives than the supergravity approximation, for example higher powers of the curvature tensors. Some of these terms have been computed from string scattering and supersymmetry, see for example [5- 9]. It is interesting to study if the hidden symmetries of the lowest order supergravity action are preserved by these higher order derivative corrections.

Recently this question has been studied, with results mostly suggesting that the hidden symmetries are broken by higher order in derivatives corrections [10-13]. The purpose of this note is to show that at least in the case of pure gravity reduced from four to three space-time dimensions the $R^{2}$ extension to fourth order in derivatives does not affect the symmetry enhancement. One argument for the breakdown of the symmetry has been the appearance of dilaton pre-factors in the reduced higher derivative correction terms which seem to spoil the invariance under the enhanced symmetry groups since dilaton scaling 
is one of the hidden symmetry transformations. However, working in the context of a perturbative $\alpha^{\prime}$ formulation we find here that the transformation laws themselves can be modified at next order in $\alpha^{\prime}$ in precisely the right way to restore the invariance. The case treated here is very simple, and has no good string theory origin, but can be seen as a first step towards the more complicated cases arising in string theory and serves to illustrate a mechanism for preserving the hidden symmetries in the presence of higher derivative terms. Our analysis is similar in spirit to that of 14, 15].

This note is structured as follows. In section 2 we study pure gravity in four dimensions extended to fourth order in derivatives and show that in this case the most general such correction (expressed in terms of the Riemann and Ricci tensors) is actually trivial in the sense that it can be made to vanish by means of local field redefinitions. This implies in particular that it cannot break Ehlers symmetry. We then proceed to consider the reduction of the $R^{2}$ terms in general and exhibit explicitly a large set of field redefinitions that effectively eliminate the higher derivative correction and restore the explicit Ehlers symmetry of the action. In section 1 we discuss the modified $\operatorname{SL}(2, \mathbb{R})$ action on the fields, in particular the subset of global scaling transformations, and explain how the problem of different scaling is resolved in this case such that the symmetry is restored. Finally, in section 5 we offer some comments on the general case which is currently under investigation. In the appendix we list the curvature tensors that arise in the reduction.

\section{2. $R^{2}$ corrected action in $(3+1)$ dimensions}

Pure gravity in a four-dimensional space-time has a hidden $\operatorname{SL}(2, \mathbb{R})$ symmetry [4]. It is exhibited by performing a dimensional reduction of the theory to three dimensions and dualising the degree of freedom of the obtained Kaluza-Klein vector to a scalar. This scalar, called the axion, together with the dilaton obtained also from the dimensional reduction, parametrizes the coset space $\mathrm{SL}(2, \mathbb{R}) / \mathrm{SO}(2)$. We are interested in investigating if this hidden symmetry is affected by higher derivative corrections.

Our starting point is then the Einstein-Hilbert action in four dimensions and its correction by fourth order in derivative terms. The most general such action that one can write is ${ }^{1}$

$$
\hat{S}=\int d^{4} x \hat{E}\left[\hat{R}+\alpha^{\prime}\left(\mathbf{m}_{1} \hat{R}^{M N P Q} \hat{R}_{M N P Q}+\mathbf{m}_{2} \hat{R}^{M N} \hat{R}_{M N}+\mathbf{m}_{3} \hat{R}^{2}\right)\right],
$$

with $\left\{\mathbf{m}_{i}\right\}$ three arbitrary real constants and $\alpha^{\prime}$ a dimensionful parameter, with dimensions (Length $)^{2}$. Hatted objects and capital letters denote four-dimensional quantities while small characters are generally reserved for three-dimensional quantities. We work in a vielbein formalism so that $\sqrt{-\hat{G}}=\operatorname{det}\left(\hat{E}_{M}{ }^{A}\right)=: \hat{E}$.

To see that the correction to the Einstein-Hilbert action in four dimensions is trivial, one uses field redefinitions of the metric and the fact that the Gauss-Bonnet combination

$$
\hat{R}_{G B}=\hat{R}^{M N P Q} \hat{R}_{M N P Q}-4 \hat{R}^{M N} \hat{R}_{M N}+\hat{R}^{2},
$$

\footnotetext{
${ }^{1}$ We do not consider the self-dual and anti-self-dual parts of the Weyl tensor separately but only the combination in which they appear in the Riemann tensor since this is the expression which generalizes to higher dimensions.
} 
is a topological invariant in four dimensions and does not contribute to the dynamics. This fact can be used to eliminate the correction term proportional to the contraction of the Riemann tensor with itself by subtracting from the action (2.1) a term proportional to the Gauss-Bonnet combination that cancels it. The remaining fourth order contributions can be eliminated by performing the following field redefinition of the four-dimensional metric

$$
\hat{G}_{M N} \longrightarrow \hat{G}_{M N}+\alpha^{\prime} \delta \hat{G}_{M N}=\hat{G}_{M N}+\alpha^{\prime}\left(\mathbf{n}_{1} \hat{R}_{M N}+\mathbf{n}_{2} \hat{G}_{M N} \hat{R}\right),
$$

with $\mathbf{n}_{1}=-\left(4 \mathbf{m}_{1}+\mathbf{m}_{2}\right)$ and $\mathbf{n}_{2}=\left(\mathbf{m}_{1}+\frac{1}{2} \mathbf{m}_{2}+\mathbf{m}_{3}\right)$. The action in the redefined metric is simply the original Einstein-Hilbert action and therefore there is a choice of fields after dimensional reduction such that the Ehlers symmetry of (2.1) is preserved. Instead of using this field basis, we will continue considering the action (2.1) and perform explicitly its dimensional reduction to three dimensions, as an example of the more general computation for higher dimensions and as a tool to describing the resolution to the problematic different scaling properties of the various orders in $\alpha^{\prime}$ below. In the higher-dimensional case the Gauss-Bonnet combination is no longer a topological invariant and one cannot use it to eliminate a term in the correction and the action (2.1) is no longer equivalent to the Einstein-Hilbert action. There are, however, simplifications to the problem that occur in the compactification to three dimensions in the general case. For the reduction from four to three dimensions further simplifications arise since the local hidden symmetry $\mathrm{SO}(2)$ is abelian.

\section{The reduced corrected action}

We consider now the dimensional reduction of the corrected Einstein-Hilbert action (2.1) to three dimensions along a space-like direction. The coordinates split as $x^{M}=\left(x^{m}, \tilde{z}\right)$, $M=0, \ldots, 3, m=0, \ldots, 2$ with $\tilde{z}$ the compact direction. Our ansatz for the reduction of the four-dimensional metric is

$$
d \hat{s}^{2}=e^{-\phi} d s^{2}+e^{\phi}\left(d \tilde{z}+A_{m} d x^{m}\right)^{2} .
$$

The three-dimensional metric is $d s^{2}=g_{m n} d x^{m} d x^{n}$ and the dilaton dependence in this ansatz has been chosen such that the reduced action is in Einstein frame with standard normalization for the terms in the lowest order reduced action. Our results are independent of this choice but we adopt it for convenience. The reduction of the corrected gravity action (2.1) gives the following three-dimensional action

$$
\begin{aligned}
S=\int d^{3} x e & \left\{R-\frac{1}{2}(\partial \phi)^{2}-\frac{1}{4} e^{2 \phi} F^{2}\right. \\
+\alpha^{\prime} & {\left[e ^ { \phi } \left(\left(-\mathbf{m}_{1}+\mathbf{m}_{3}\right) R^{2}+\left(4 \mathbf{m}_{1}+\mathbf{m}_{2}\right) R^{m n} R_{m n}\right.\right.} \\
& -\left(4 \mathbf{m}_{1}+\mathbf{m}_{2}\right) R^{m n} \partial_{m} \phi \partial_{n} \phi-\left(2 \mathbf{m}_{1}+\mathbf{m}_{2}+2 \mathbf{m}_{3}\right) D^{m} R \partial_{m} \phi \\
& -\left(\mathbf{m}_{1}+\mathbf{m}_{2}+3 \mathbf{m}_{3}\right) R(\partial \phi)^{2}-\left(3 \mathbf{m}_{1}+\mathbf{m}_{2}+\mathbf{m}_{3}\right) \partial^{m} \phi \nabla_{m}(\square \phi) \\
& \left.-\left(4 \mathbf{m}_{1}+\frac{3}{2} \mathbf{m}_{2}+2 \mathbf{m}_{3}\right) \square \phi(\partial \phi)^{2}+\frac{1}{4}\left(3 \mathbf{m}_{1}+\mathbf{m}_{2}+\mathbf{m}_{3}\right)(\partial \phi)^{4}\right)
\end{aligned}
$$




$$
\begin{aligned}
+e^{3 \phi} & \left(-\left(6 \mathbf{m}_{1}+\frac{3}{2} \mathbf{m}_{2}\right) R^{m n} F_{m p} F_{n}{ }^{p}+\frac{1}{2}\left(3 \mathbf{m}_{1}+\frac{1}{2} \mathbf{m}_{2}-\mathbf{m}_{3}\right) R F^{2}\right. \\
& -\left(\mathbf{m}_{1}+\frac{1}{4} \mathbf{m}_{2}\right) F^{m n} \square F_{m n}-\frac{1}{2}\left(4 \mathbf{m}_{1}+\mathbf{m}_{2}\right) F^{m p} F_{p}^{n} \nabla_{m} \partial_{n} \phi \\
& +\left(4 \mathbf{m}_{1}+\mathbf{m}_{2}\right) F^{m p} F_{p}^{n} \partial_{m} \phi \partial_{m} \phi-\frac{1}{8}\left(16 \mathbf{m}_{1}+5 \mathbf{m}_{2}+4 \mathbf{m}_{3}\right) F^{2} \square \phi \\
& \left.\left.\left.+\frac{1}{4}\left(5 \mathbf{m}_{1}+\frac{3}{2} \mathbf{m}_{2}+\mathbf{m}_{3}\right) F^{2}(\partial \phi)^{2}\right)+e^{5 \phi} \frac{1}{16}\left(11 \mathbf{m}_{1}+3 \mathbf{m}_{2}+\mathbf{m}_{3}\right)\left(F^{2}\right)^{2}\right]\right\} .
\end{aligned}
$$

The curvature tensors and covariant derivatives are constructed from the three-dimensional metric $g_{m n}$. The complete decomposition of the four-dimensional curvature tensors in terms of the three-dimensional fields is given for completeness in the appendix. To obtain the expresion (3.2) we have used Bianchi identities and the special properties of the three-dimensional space-time and of the abelian gauge transformations of the Kaluza-Klein vector in addition to some integrations by parts on the action obtained directly using the dimensional reduction ansatz (3.1). These manipulations are convenient because in (3.2) all order $\alpha^{\prime}$ terms vanish for the Gauss-Bonnet values, illustrating the fact that they constitute a total derivative.

As mentioned before the lowest order terms in (3.2) possess a hidden $\mathrm{SL}(2, \mathbb{R})$ symmetry. To observe it one has to dualise the Kaluza-Klein vector field strength to the gradient of a scalar field $\chi$, the axion. This can be done by the introduction of a Lagrange multiplier term $\int e \frac{1}{2} \epsilon^{m n p} \partial_{m} \chi F_{n p}$ exchanging the Bianchi identity of the antisymmetric tensor field for the equation of motion of the axion field $\chi$. One can then consider $F_{m n}$ rather than $A_{m}$ as an independent field and eliminate it in favour of $\chi$ by solving its equation of motion which is only algebraic. The presence of higher order corrections will affect the duality relation but in a perturbative analysis one can use at each order in $\alpha^{\prime}$ the lower order solution. Additionally, at first order in $\alpha^{\prime}$ the corrections to the duality relation cancel out in the action, so that we can safely use the lowest order relation to exchange the two fields. The lowest order action can then be written in an explicitly symmetric way by formulating it as a non-linear $\sigma$-model on the coset space $\mathrm{SL}(2, \mathbb{R}) / \mathrm{SO}(2)$ parametrized by the dilaton and axion. In terms of the Cartan-Killing trace it is given by

$$
\tilde{S}_{0}=\int d^{3} x e\left(R-\operatorname{tr}\left(P^{m} P_{m}\right)\right)=\int d^{3} x e\left(R-\frac{1}{2}(\partial \phi)^{2}-\frac{1}{2} e^{-2 \phi}(\partial \chi)^{2}\right) .
$$

Here $P_{m}$ is the projection along the coset directions of the Cartan form whose parametrization in terms of the scalar fields is given by

$$
P_{m}=\left(\begin{array}{cc}
\frac{1}{2} \partial_{m} \phi & \frac{1}{2} e^{-\phi} \partial_{m} \chi \\
\frac{1}{2} e^{-\phi} \partial_{m} \chi & -\frac{1}{2} \partial_{m} \phi
\end{array}\right) .
$$

The SL $(2, \mathbb{R})$ transformations only act on the scalar sector leaving the three-dimensional metric and curvature tensors invariant.

The first order in $\alpha^{\prime}$ terms in (3.2) seem hard to reconcile with this symmetry. However, they can be brought into a symmetric form by performing redefinitions on the fields, as 
was also done in [14]. We consider the following general fourth order action exhibiting explicitly Ehlers symmetry,

$$
\begin{aligned}
\tilde{S}_{s}= & \int d^{3} x e\left[R-\operatorname{tr}\left(P^{m} P_{m}\right)+\alpha^{\prime}\left(a \operatorname{tr}\left(P^{m} P_{m}\right)^{2}+b \operatorname{tr}\left(P^{m} P^{n} P_{m} P_{n}\right)+c R_{c}^{2}\right)\right] \\
= & \int d^{3} x e\left\{R-\frac{1}{2}(\partial \phi)^{2}-\frac{1}{2} e^{-2 \phi}(\partial \chi)^{2}\right. \\
& \left.+\alpha^{\prime}\left[(a+b)\left((\partial \phi)^{4}+2 e^{-2 \phi}(\partial \phi)^{2}(\partial \chi)^{2}+\frac{1}{4} e^{-4 \phi}(\partial \chi)^{4}\right)+4 b e^{-2 \phi} \partial_{a} \phi \partial_{b} \phi \partial^{a} \chi \partial^{b} \chi+c R_{c}^{2}\right]\right\} .
\end{aligned}
$$

Here $a, b$ and $c$ are arbitrary real constants and the $R_{c}^{2}$ term refers to any pure curvature fourth order in derivatives terms, the contractions are suppressed for simplicity. ${ }^{2}$ Expressed in terms of the graviphoton field strength and up to fourth order in derivatives the action (3.5) becomes

$$
\begin{aligned}
& S_{s}=\int d^{3} x e\left\{R-\frac{1}{2}(\partial \phi)^{2}-\frac{1}{4} e^{2 \phi} F^{2}+\alpha^{\prime}\left[( a + b ) \left((\partial \phi)^{4}-e^{2 \phi}(\partial \phi)^{2} F^{2}\right.\right.\right. \\
& \left.\left.\left.+\frac{1}{4} e^{4 \phi}\left(F^{2}\right)^{2}\right)+4 b e^{2 \phi} F^{m p} F_{p}^{n} \partial_{m} \phi \partial_{n} \phi+c R_{c}^{2}\right]\right\} \text {. }
\end{aligned}
$$

On this action we perform the following class of field redefinitions

$$
\begin{aligned}
\delta \phi= & a_{1}(\partial \phi)^{2}+a_{2} \square \phi+a_{3} R+a_{4} F^{2}, \\
\delta F_{m n}= & b_{1} \square F_{m n}+b_{2} F^{l}{ }_{[n} R_{m] l}+b_{3} F^{l}{ }_{[n} \partial_{m]} \phi \partial_{l} \phi+b_{4} F^{l}{ }_{[n} \nabla_{m]} \partial_{l} \phi \\
& +b_{5} F_{m n}(\partial \phi)^{2}+b_{6} F_{m n} \square \phi+b_{7} F_{m n} R+b_{8} F_{m n} F^{2}, \\
\delta g_{m n}= & c_{1} R_{m n}+c_{2} \partial_{m} \phi \partial_{n} \phi+c_{3} \nabla_{m} \partial_{n} \phi+c_{4} F_{m p} F_{n}{ }^{p}+ \\
& +g_{m n}\left(d_{1}(\partial \phi)^{2}+d_{2} \square \phi+d_{3} R+d_{4} F^{2}\right) .
\end{aligned}
$$

The coefficients $\left\{a_{i}, b_{i}, c_{i}, d_{i}\right\}$ are real parameters and may have a dilaton dependence in the form of an exponential prefactor. These are the most general field redefinitions that produce terms in the action like the ones appearing in the reduced higher order action (3.2). The redefinition of $F_{m n}$ is not integrable to a local field redefinition of the underlying vector potential $A_{m}$ for all values of the coefficients $b_{i}$. However, it will turn out below that there are choices for $b_{i}$ such that the symmetry is preserved and that an interpretation in terms of a local redefinition of $A_{m}$ is possible.

Under these redefinitions the symmetric action (3.6) gets modified at non-zero orders

\footnotetext{
${ }^{2}$ For bigger symmetry groups, like the ones arising when compactifying gravity in more than four dimensions down to three, there can be four independent contributions of quartic traces, with $\operatorname{tr}\left(P^{m} P_{m} P^{n} P_{n}\right)$ and $\operatorname{tr}\left(P^{m} P^{n}\right) \operatorname{tr}\left(P_{m} P_{n}\right)$ apparently missing in 3.5$)$. However, in the case of $\mathrm{SL}(2, \mathbb{R})$ only two of these structures are linearly independent. We also disregard terms of the form $\operatorname{tr}\left(P^{m} P_{m}\right) R$ or $\operatorname{tr}\left(P^{m} P^{n}\right) R_{m n}$ since they are not needed here and they have a different scaling behaviour.
} 
in $\alpha^{\prime}$ to become, to first order

$$
\begin{aligned}
S_{s}= & S_{0}+\alpha^{\prime} \int d^{3} x e\left\{(a+b)\left((\partial \phi)^{4}-e^{2 \phi}(\partial \phi)^{2} F^{2}+\frac{1}{4} e^{4 \phi}\left(F^{2}\right)^{2}\right)\right. \\
& +4 b e^{2 \phi} F^{m p} F^{n}{ }_{p} \partial_{m} \phi \partial_{n} \phi+c R_{c}^{2}-\frac{1}{2} e^{2 \phi} F^{m n} \delta F_{m n}-\left(\frac{1}{2} e^{2 \phi} F^{2}-\square \phi\right) \delta \phi \\
& \left.-\delta g_{m n}\left[R^{m n}-\frac{1}{2} g^{m n}\left(R-\frac{1}{2}(\partial \phi)^{2}-\frac{1}{4} e^{2 \phi} F^{2}\right)-\frac{1}{2} \partial^{m} \phi \partial^{n} \phi-\frac{1}{2} e^{2 \phi} F^{m p} F_{p}^{n}\right]\right\} .
\end{aligned}
$$

Inserting the redefinitions (3.7) and performing some integrations by parts brings (3.6) into the form of the action (3.2) allowing one to compare the coefficients in both expressions term by term, which results in a system of linear equations for the arbitrary coefficients in (3.7) in terms of $\left\{\phi, \mathbf{m}_{1}, \mathbf{m}_{2}, \mathbf{m}_{3}\right\}$. The system turns out to be solvable for arbitrary values of $\mathbf{m}_{1}, \mathbf{m}_{2}$ and $\mathbf{m}_{3}$, as was expected from the general considerations of the previous section. This is sufficient to show that Ehlers symmetry is not broken by a general order $\alpha^{\prime}$ correction to the Einstein-Hilbert action.

The actual solution is not unique, there turns out to be some arbitrariness in the choice of some of the coefficients. The general result is

$$
\begin{aligned}
\delta \phi= & {\left[\frac{1}{2} c_{3}+\frac{1}{4} d_{2}-\frac{1}{2} e^{\phi}\left(2 \mathbf{m}_{1}+\mathbf{m}_{2}+2 \mathbf{m}_{3}\right)\right](\partial \phi)^{2}+e^{\phi}\left(3 \mathbf{m}_{1}+\mathbf{m}_{2}+\mathbf{m}_{3}\right) \square \phi } \\
& +\left[-\frac{1}{2} d_{2}+e^{\phi}\left(2 \mathbf{m}_{1}+\mathbf{m}_{2}+2 \mathbf{m}_{3}\right)\right] R+a_{4} F^{2}, \\
\delta F_{m n}= & \frac{1}{2} e^{\phi}\left(4 \mathbf{m}_{1}+\mathbf{m}_{2}\right) \square F_{m n}+e^{-2 \phi}\left[-2 c_{4}+2 e^{3 \phi}\left(4 \mathbf{m}_{1}+\mathbf{m}_{2}\right)\right] F^{l}{ }_{[n} R_{m] l} \\
& +e^{-2 \phi}\left[e^{2 \phi} c_{3}+c_{4}+8 e^{2 \phi} b-\frac{3}{2} e^{3 \phi}\left(4 \mathbf{m}_{1}+\mathbf{m}_{2}\right)\right] F_{[n}^{l} \partial_{m]} \phi \partial_{l} \phi \\
& +\left[c_{3}+e^{\phi}\left(4 \mathbf{m}_{1}+\mathbf{m}_{2}\right)\right] F^{l}{ }_{[n} \nabla_{m]} \partial_{l} \phi+e^{-2 \phi}\left[-\frac{3}{4} e^{2 \phi} c_{3}-\frac{1}{4} e^{2 \phi} d_{2}-\frac{1}{2} c_{4}-\frac{1}{2} d_{4}\right. \\
& \left.-\frac{1}{4} e^{3 \phi}\left(9 \mathbf{m}_{1}+2 \mathbf{m}_{2}-\mathbf{m}_{3}\right)\right] F_{m n}(\partial \phi)^{2} \\
& +\frac{1}{4}\left[-c_{3}+d_{2}+8 e^{-2 \phi} a_{4}+e^{\phi}\left(4 \mathbf{m}_{1}+\mathbf{m}_{2}\right)\right] F_{m n} \square \phi \\
& +\frac{1}{2} e^{-2 \phi}\left[e^{2 \phi} d_{2}+2 c_{4}+2 d_{4}-e^{3 \phi}\left(7 \mathbf{m}_{1}+2 \mathbf{m}_{2}+\mathbf{m}_{3}\right)\right] F_{m n} R \\
& +\frac{1}{4}\left[-4 a_{4}+c_{4}+d_{4}-\frac{1}{2} e^{3 \phi}\left(11 \mathbf{m}_{1}+3 \mathbf{m}_{2}+\mathbf{m}_{3}\right)\right] F_{m n} F^{2}, \\
\delta g_{m n}= & -e^{\phi}\left(4 \mathbf{m}_{1}+\mathbf{m}_{2}\right) R_{m n}+\left[c_{3}+\frac{1}{2} e^{\phi}\left(4 \mathbf{m}_{1}+\mathbf{m}_{2}\right)\right] \partial_{m} \phi \partial_{n} \phi+c_{3} D_{m} \partial_{n} \phi+c_{4} F_{m p} F_{n}^{p} \\
+ & g_{m n}\left[-\frac{1}{2} e^{\phi}\left(2 \mathbf{m}_{1}+\mathbf{m}_{2}+2 \mathbf{m}_{3}\right)(\partial \phi)^{2}+d_{2} \square \phi+e^{\phi}\left(2 \mathbf{m}_{1}+\mathbf{m}_{2}+2 \mathbf{m}_{3}\right) R+d_{4} F^{2}\right], \\
a= & -b, \quad c=0 .
\end{aligned}
$$

The six parameters $\left(b, a_{4}, c_{3}, c_{4}, d_{2}, d_{4}\right)$ are free, the solution is highly degenerate. For consistency one expects that the particular field redefinition (2.3) which eliminated the 
whole correction term at the four-dimensional level should be an allowed solution. In fact, one can compute the redefinitions on the three-dimensional fields induced by (2.3) after performing the dimensional reduction and show that these correspond to (3.9) for a particular choice of the six free parameters given by

$$
\begin{aligned}
& a_{4}=-\frac{1}{4} e^{3 \phi}\left(5 \mathbf{m}_{1}+\frac{3}{2} \mathbf{m}_{2}+\mathbf{m}_{3}\right), c_{3}=0, c_{4}=\frac{1}{2} e^{3 \phi}\left(4 \mathbf{m}_{1}+\mathbf{m}_{2}\right), \\
& d_{2}=e^{\phi}\left(2 \mathbf{m}_{1}+\mathbf{m}_{2}+2 \mathbf{m}_{3}\right), d_{4}=-\frac{1}{2} e^{3 \phi}\left(3 \mathbf{m}_{1}+\mathbf{m}_{2}+\mathbf{m}_{3}\right), b=0 .
\end{aligned}
$$

For this choice the correction $\delta F_{m n}$ can be traced back to a correction to the Kaluza-Klein vector potential $A_{m}$.

Now that we have proved that the fourth order correction does not affect the hidden symmetry of the action, we will describe in some more detail the action of a subset of the $\mathrm{SL}(2, \mathbb{R})$ transformations and show how they are modified by fourth order terms.

\section{Corrected $\operatorname{SL}(2, \mathbb{R})$ transformations}

At lowest order the Ehlers $\mathrm{SL}(2, \mathbb{R})$ transformations include scaling transformations of the fields, $\phi \longrightarrow \phi+\sigma, \chi \longrightarrow e^{\sigma} \chi$, associated with the Cartan generator of $\mathrm{SL}(2, \mathbb{R})$. One can then read off the transformation of the four-dimensional curvature objects from their expresions in terms of the lower-dimensional fields obtained when performing the dimensional reduction. One finds that the scalar curvature and four-dimensional measure transforms under this lowest order global rescaling as (cf. formulas in the appendix)

$$
\hat{R} \longrightarrow e^{\sigma} \hat{R}, \quad \hat{E}=e^{-\phi} e \longrightarrow e^{-\sigma} \hat{E} .
$$

Clearly the combination $\hat{E} \hat{R}$ is then invariant, however, the higher order contribution $\hat{E} \hat{R}^{2}$ is not invariant and therefore seems to break the $\mathrm{SL}(2, \mathbb{R})$ symmetry. This presents an apparent contradiction with our previous results where we showed that the symmetry is preserved by general $R^{2}$ order curvature corrections and, moreover, that these are trivial in this case.

The problem arises from the appearance of overall exponential prefactors of the dilaton carried by the expansion of the higher dimensional curvature objects after dimensional reduction, as is evident from the explicit results in the appendix. At lowest order these prefactors can be made to cancel out with the ones coming from the reduction of the measure by choosing an appropriate frame, most conveniently the Einstein frame as in (4.1). However, then they will not cancel for the higher order curvature terms. This is a generic feature of the dimensional reduction of supergravity actions and is related to the observation in [16, 10, 17] according to which higher curvature terms are associated with the weights of the hidden symmetry algebra rather than its roots which occur in the usual parametrization of the coset space. The dilaton prefactors are at the same time associated to the volume of the compactified space and are central when trying to complete the weight structure by an automorphic form of the discrete version of the hidden symmetry algebra [11-113]. 
However, in this simple case the symmetry as we have seen is not broken. The solution to the puzzle is that the transformation rules for the original $\phi, \chi$ and the metric $g_{m n}$ are only correct up to lowest order and change once $\alpha^{\prime}$ terms are considered, giving new contributions to the transformation of the action that restore the symmetry. To derive the modification of the transformation rules of the three-dimensional fields one needs to notice that the redefined fields, in terms of which the symmetry of the action is manifest, transform by construction just under the usual (lowest order) Ehlers transformations. The original fields then have an expansion in terms of the redefined ones. From this fact one can derive the $\alpha^{\prime}$ corrections to the transformation laws of the original fields. The result in the case of the scaling transformation is the following,

$$
\begin{aligned}
\phi & \longrightarrow \phi+\sigma+\alpha^{\prime} \delta \phi\left(1-e^{\sigma}\right), \\
F_{m n} & \longrightarrow e^{-\sigma} F_{m n}+\alpha^{\prime} \delta F_{m n}\left(e^{-\sigma}-1\right), \\
g_{m n} & \longrightarrow g_{m n}+\alpha^{\prime} \delta g_{m n}\left(1-e^{\sigma}\right) .
\end{aligned}
$$

It is easy to see that, when acting on the lowest order Einstein-Hilbert action, the $\alpha^{\prime}$ terms in the transformations (4.2) generate a term that reconstructs the original correction to the action globally rescaled by a factor of $e^{\sigma \phi}$, which precisely cancels the actual correction terms transformed at lowest order as follows from (4.1). The symmetry is then restored up to fourth order in derivatives. The other transformations that make up the $\operatorname{SL}(2, \mathbb{R})$ group can be analysed in a similar way and order $\alpha^{\prime}$ modifications to them also appear. Since the transformations of the redefined fields close to form the group $\operatorname{SL}(2, \mathbb{R})$ the $\alpha^{\prime}$ modified transformations of the original fields naturally form an $\operatorname{SL}(2, \mathbb{R})$ up to order $\alpha^{\prime}$.

\section{Outlook}

The case of four-dimensional gravity reduced to three dimensions studied in this paper contains a number of non-generic features. It is not clear to what extent the result that the hidden symmetry is unbroken by general $R^{2}$ corrections carries over to the reduction of $R^{2}$ extended $(3+n)$-dimensional gravity to three dimensions. Nevertheless we believe that the techniques used in this paper can be employed to address this question and our results emphasise that $\alpha^{\prime}$-corrections to the symmetry transformations are an important feature to be considered.

One of the special properties of $R^{2}$ corrections in $(3+1)$ dimensions is the topological nature of the Gauss-Bonnet combination which can be used to remove the square of the full Riemann tensor. The remaining terms are then proportional to the lowest order equations of motion and can be removed by field redefinitions in $(3+1)$ dimensions. This clearly is

no longer possible in the general case since $\hat{R}_{A B C D} \hat{R}^{A B C D}$ is not part of a total derivative. Furthermore, one cannot generate such a term from a field redefinition in $(3+n)$ dimensions before reduction for $n>1$. We still deem it likely that this term can be accounted for in a symmetric action by a field redefinition after reduction to three dimensions since the Weyl tensor vanishes identically and the three-dimensional Riemann tensor therefore can be expressed in terms of the Ricci tensor and scalar, both of which do arise from field 
redefinitions. First computations indicate that this can be implemented and that also the other terms arising in the reduction can be treated in a similar fashion. We plan to report on this in more detail in the near future.

String effects and in particular string loops are expected to break the hidden symmetry arising in dimensional reduction to its arithmetic version [18, 19]. This is consistent with the present analysis where we only considered perturbative $\alpha^{\prime}$ corrections to gravity. In the full theory it seems likely that automorphic forms are needed in order to maintain the discrete symmetry.

\section{Acknowledgments}

We are grateful to L. Bao, M. Cederwall, K. Meissner, H. Nicolai, B.E.W. Nilsson, K. Peeters and J. Plefka for discussions. AK would like to thank Chalmers University, Gothenburg, for its warm hospitality and support. We thank Y. Michel and B. Pioline for correspondence.

\section{A. Reduction of curvature tensors}

Here, for completeness, we list the results of the dimensional reduction of the curvature tensors from four to three dimensions derived from the general ansatz

$$
d \hat{s}^{2}=e^{2 \alpha \phi} d s^{2}+e^{2 \beta \phi}\left(d \tilde{z}+A_{m} d x^{m}\right)^{2} .
$$

Again, hatted objects and capital letters denote four-dimensional quantities while small characters are reserved for three-dimensional ones. The coordinates split as $x^{M}=$ $\left(x^{m}, \tilde{z}\right), \quad M=0, \ldots, 3, m=0, \ldots, 2$ with $\tilde{z}$ the space-like compact direction. Flat indices are denoted by letters at the beginning of the alphabet and split as $A=$ $(a, z), A=0, \ldots, 3, a=0, \ldots, 2$. They are contracted using the flat Minkowski metric $\hat{\eta}_{A B}=\operatorname{diag}(-,+,+, \ldots)$. In (A.1) $\alpha, \beta$ are arbitrary real constants, in general we choose them to have values $\alpha=-\beta=-\frac{1}{2}$ in order for the the lowest order reduced gravity action to be in the Einstein frame. The tangent space components of the curvature tensors then are

$$
\begin{aligned}
& \hat{R}_{a z b z}=e^{-2 \alpha \phi}\left[-\beta D_{a} \partial_{b} \phi+\beta(2 \alpha-\beta) \partial_{a} \phi \partial_{b} \phi-\alpha \beta \eta_{a b}(\partial \phi)^{2}+\frac{1}{4} e^{(-2 \alpha+2 \beta) \phi} F_{a c} F_{b c}\right], \\
& \hat{R}_{a z b c}=e^{(-3 \alpha+\beta) \phi}\left[-\frac{1}{2} D_{a} F_{b c}+\alpha \partial_{e} \phi F_{e[b} \eta_{c] a}+(\alpha-\beta) \partial_{a} \phi F_{b c}-(\alpha-\beta) \partial_{[b} \phi F_{c] a}\right], \\
& \hat{R}_{a b c d}=e^{-2 \alpha \phi}\left[R_{a b c d}-2 \alpha D_{a} \partial_{[c} \phi \eta_{d] b}+2 \alpha D_{b} \partial_{[c} \phi \eta_{d] a}\right. \\
& +2 \alpha^{2} \partial_{a} \phi \partial_{[c} \phi \eta_{d] b}-2 \alpha^{2} \partial_{b} \phi \partial_{[c} \phi \eta_{d] a}-2 \alpha^{2}(\partial \phi)^{2} \eta_{a[c} \eta_{d] b} \\
& \left.-\frac{1}{2} e^{(-2 \alpha+2 \beta) \phi} F_{a b} F_{c d}+\frac{1}{2} e^{(-2 \alpha+2 \beta) \phi} F_{a[c} F_{d] b}\right] \text {, } \\
& \hat{R}_{z z}=e^{-2 \alpha \phi}\left[-\beta \square \phi-\left(\beta^{2}+\alpha \beta\right)(\partial \phi)^{2}+\frac{1}{4} e^{(-2 \alpha-2 \beta) \phi} F^{2}\right],
\end{aligned}
$$




$$
\begin{aligned}
\hat{R}_{z a}=-\frac{1}{2} e^{(-3 \alpha+\beta) \phi}\left[D^{c} F_{c a}+(-\alpha+3 \beta) \partial_{c} \phi F_{c a}\right], & \\
\hat{R}_{a b}=e^{-2 \alpha \phi} & {\left[R_{a b}-(\alpha+\beta) D_{a} \partial_{b} \phi+\left(2 \alpha \beta-\beta^{2}+\alpha^{2}\right) \partial_{a} \phi \partial_{b} \phi-\alpha \eta_{a b} \square \phi\right.} \\
& \left.\quad-\left(\alpha^{2}+\alpha \beta\right) \eta_{a b}(\partial \phi)^{2}-\frac{1}{2} e^{(-2 \alpha+2 \beta) \phi} F_{a c} F_{b c}\right], \\
\hat{R}=e^{-2 \alpha \phi} & {\left[R-2(2 \alpha+\beta) \square \phi-\frac{1}{4} e^{(-2 \alpha+2 \beta) \phi} F^{2}-2\left(\alpha \beta+\alpha^{2}+\beta^{2}\right)(\partial \phi)^{2}\right] . }
\end{aligned}
$$

Here, $D_{a}$ is the $\mathrm{SO}(1,2)$ Lorentz covariant derivative in three dimensions, e.g. $D_{a} \partial_{b} \phi=$ $\partial_{a} \partial_{b} \phi+\omega_{a b}^{c} \partial_{c} \phi$. A good review on the subject of dimensional reduction can be found in [20].

\section{References}

[1] E. Cremmer and B. Julia, The $N=8$ supergravity theory. 1. the lagrangian, Phys. Lett. B 80 (1978) 48 .

[2] E. Cremmer, B. Julia, H. Lü and C.N. Pope, Dualisation of dualities. i, Nucl. Phys. B 523 (1998) 73 hep-th/9710119.

[3] E. Cremmer, B. Julia, H. Lü and C.N. Pope, Higher-dimensional origin of $D=3$ coset symmetries, hep-th/9909099.

[4] J. Ehlers, Dissertation, Hamburg University (1957).

[5] K. Peeters, P. Vanhove and A. Westerberg, Supersymmetric higher-derivative actions in ten and eleven dimensions, the associated superalgebras and their formulation in superspace, Class. and Quant. Grav. 18 (2001) 843 hep-th/0010167.

[6] R. Iengo, Computing the $R^{4}$ term at two super-string loops, JHEP 02 (2002) 035 hep-th/0202058.

[7] M. Cederwall, U. Gran, B.E.W. Nilsson and D. Tsimpis, Supersymmetric corrections to eleven-dimensional supergravity, JHEP 05 (2005) 052 hep-th/0409107.

[8] M.B. Green and P. Vanhove, Duality and higher derivative terms in M-theory, JHEP 01 (2006) 093 hep-th/0510027.

[9] G. Policastro and D. Tsimpis, $R^{4}$, purified, Class. and Quant. Grav. 23 (2006) 4753 hep-th/0603165.

[10] N. Lambert and P. West, Enhanced coset symmetries and higher derivative corrections, Phys. Rev. D 74 (2006) 065002 hep-th/0603255.

[11] N. Lambert and P. West, Duality groups, automorphic forms and higher derivative corrections, Phys. Rev. D 75 (2007) 066002 hep-th/0611318.

[12] L. Bao, M. Cederwall and B.E.W. Nilsson, Aspects of higher curvature terms and U-duality, arXiv:0706.1183.

[13] Y. Michel and B. Pioline, Higher derivative corrections, dimensional reduction and Ehlers duality, arXiv:0706.1769. 
[14] K.A. Meissner, Symmetries of higher-order string gravity actions, Phys. Lett. B 392 (1997) 298 hep-th/9610131.

[15] N. Kaloper and K.A. Meissner, Duality beyond the first loop, Phys. Rev. D 56 (1997) 7940 hep-th/9705193.

[16] T. Damour and H. Nicolai, Higher order M-theory corrections and the Kac-Moody algebra $E_{10}$, Class. and Quant. Grav. 22 (2005) 2849 hep-th/0504153.

[17] T. Damour, A. Hanany, M. Henneaux, A. Kleinschmidt and H. Nicolai, Curvature corrections and Kac-Moody compatibility conditions, Gen. Rel. Grav. 38 (2006) 1507 hep-th/0604143.

[18] C.M. Hull and P.K. Townsend, Unity of superstring dualities, Nucl. Phys. B 438 (1995) 109 hep-th/9410167.

[19] N.A. Obers and B. Pioline, U-duality and M-theory, Phys. Rept. 318 (1999) 113 hep-th/9809039.

[20] C.N. Pope, Kaluza-Klein theory, available online under the URL: http://faculty.physics.tamu.edu/pope/ihplec.pdt. 